\title{
Einstein Manifolds and Extremal Kähler Metrics
}

\author{
Claude LeBrun* \\ SUNY Stony Brook
}

\begin{abstract}
In joint work with Chen and Weber [7], the author has elsewhere shown that $\mathbb{C P}_{2} \# 2 \overline{\mathbb{C P}}_{2}$ admits an Einstein metric. The present paper gives a new and rather different proof of this fact. Our results include new existence theorems for extremal Kähler metrics, and these allow one to prove the above existence statement by deforming the KählerEinstein metric on $\mathbb{C P}_{2} \# 3 \overline{\mathbb{C P}}_{2}$ until bubbling-off occurs.
\end{abstract}

\section{Introduction}

Recall that a Riemannian metric is said to be Einstein iff it has constant Ricci curvature [3]. When this happens, the constant value assumed by the Ricci curvature is called the Einstein constant. A fundamental problem of global Riemannian geometry is to determine precisely which smooth compact manifolds admit Einstein metrics.

While we still remain quite far from being able to determine precisely which smooth compact 4-manifolds admit Einstein metrics, notable progress has recently been achieved regarding narrower versions of the problem. For example, it was shown in [7] that if $M^{4}$ is the underlying smooth oriented manifold of a compact complex surface, then $M$ admits an Einstein metric with positive Einstein constant iff it is diffeomorphic to a Del Pezzo surface. In other words, the only allowed diffeotypes are $S^{2} \times S^{2}$ and $\mathbb{C P}_{2} \# k \overline{\mathbb{C P}}_{2}$, with

\footnotetext{
*Supported in part by NSF grant DMS-0905159.
} 
$0 \leq k \leq 8$; and, conversely, each of these candidates does actually admit an Einstein metric with positive Einstein constant.

The existence direction of the above assertion is proved by means of Kähler geometry. For example, the theory of the complex Monge-Ampère equation can be used to show that all but two of the above candidates admit Kähler-Einstein metrics [24, 25, 26]. However, $\mathbb{C P}_{2} \# \overline{\mathbb{C P}}_{2}$ and $\mathbb{C P}_{2} \# 2 \overline{\mathbb{C P}}_{2}$ cannot admit Kähler-Einstein metrics, owing to the non-reductive nature of their automorphism groups [20]. Nonetheless, Page [21] was able to construct an explicit cohomogeneity-one Einstein metric on $\mathbb{C P}_{2} \# \overline{\mathbb{C P}}_{2}$, and Derdziński [10] subsequently discovered that this metric is actually conformally Kähler. Following this lead, Chen, Weber, and the present author later proved [7] that $\mathbb{C P}_{2} \# 2 \overline{\mathbb{C P}}_{2}$ also admits a conformally Kähler, Einstein metric, although, in contrast to the the Page metric, we do not know its explicit form.

The present article will provide a new proof for the existence of an Einstein metric on $\mathbb{C P}_{2} \# 2 \overline{\mathbb{C P}}_{2}$. In the process, we will actually give a direct proof of the following, ostensibly stronger statement:

Theorem A There is a conformally Kähler, Einstein metric $h$ on $M=$ $\mathbb{C P}_{2} \# 2 \overline{\mathbb{C P}}_{2}$ for which the conformally related Kähler metric $g$ minimizes the $L^{2}$-norm of the scalar curvature among all Kähler metrics on $M$. In other words, $h$ is an absolute minimizer of the Weyl functional among all conformally Kähler metrics on $M$.

For a definition of the Weyl functional, see $\$ 3$ below. Notice that Theorem $\mathrm{A}$ does not actually assert that the relevant Einstein metric actually coincides with the metric of [7]. However, this is actually true, as a consequence of uniqueness results recently proved in [17].

Another main purpose of the present article is to prove new existence results for extremal Kähler metrics. On any toric Del Pezzo surface, we show, in Theorems 1 and 2 below, that any Kähler class in a large, specific neighborhood of $c_{1}$ is represented by an extremal Kähler metric. This not only implies Theorem A, but also allows us to prove the following:

Theorem B Let $g$ be the conformally Einstein, Kähler metric on $\mathbb{C P}_{2} \# 2 \overline{\mathbb{C P}}_{2}$ discussed in Theorem $\underline{A}$. Then there is a 1-parameter family $g_{t}, t \in[0,1)$ of extremal Kähler metrics on $\mathbb{C P}_{2} \# 3 \overline{\mathbb{C P}}_{2}$, such that $g_{0}$ is Kähler-Einstein, and such that $g_{t_{j}} \rightarrow g$ in the Gromov-Hausdorff sense for some $t_{j} \nearrow 1$. 
The approach that will be developed here hinges on a systematic study of the squared $L^{2}$-norm

$$
\mathcal{C}(g)=\int_{M} s_{g}^{2} d \mu_{g}
$$

of the scalar curvature, restricted to the space of Kähler metrics. An important variational problem for this functional was first studied by Calabi [4, 5], who constrained $g$ to only vary in a fixed Kähler class $[\omega] \in H^{2}(M, \mathbb{R})$. Calabi called the critical metrics of his restricted problem extremal Kähler metrics, and showed that the relevant Euler-Lagrange equations are equivalent to requiring that $\nabla^{1,0} s$ be a holomorphic vector field. In fact, every extremal Kähler metric turns out to be an absolute minimizer for the Calabi problem, and the proof of this [6] moreover implies the sharp estimate

$$
\frac{1}{32 \pi^{2}} \int_{M} s_{g}^{2} d \mu_{g} \geq \frac{\left(c_{1} \cdot \Omega\right)^{2}}{\Omega^{2}}+\frac{1}{32 \pi^{2}}\|\mathfrak{F}(\Omega)\|^{2}
$$

with equality iff $g$ is an extremal Kähler metric. Here

$$
\mathfrak{F}(\Omega): H^{0}\left(M, \mathcal{O}\left(T^{1,0} M\right)\right) \rightarrow \mathbb{C}
$$

denotes the Futaki invariant, and the relevant norm is the one induced by the $L^{2}$-norm on the space of holomorphy potentials [12]. In particular, for any extremal Kähler metric $g$ with Kähler class $\Omega$, one has

$$
\begin{aligned}
\int_{M} s_{0}^{2} d \mu_{g} & =32 \pi^{2} \frac{\left(c_{1} \cdot \Omega\right)^{2}}{\Omega^{2}} \\
\int_{M}\left(s-s_{0}\right)_{g}^{2} d \mu_{g} & =\|\mathfrak{F}(\Omega)\|^{2}
\end{aligned}
$$

where

$$
s_{0}=f_{M} s d \mu_{g}=\frac{\int s d \mu_{g}}{\int d \mu_{g}}
$$

denotes the average scalar curvature. Thus, letting $\mathcal{K} \subset H^{2}(M, \mathbb{R})$ be the Kähler cone of $(M, J)$, we are led to consider the action function $\mathcal{A}: \mathcal{K} \rightarrow \mathbb{R}$ defined by

$$
\mathcal{A}(\Omega)=\frac{\left(c_{1} \cdot \Omega\right)^{2}}{\Omega^{2}}+\frac{1}{32 \pi^{2}}\|\mathfrak{F}\|^{2}
$$

which we write schematically as

$$
\mathcal{A}(\Omega)=\mathcal{T}(\Omega)+\mathcal{B}(\Omega)
$$


where

$$
\mathcal{T}(\Omega)=\frac{\left(c_{1} \cdot \Omega\right)^{2}}{\Omega^{2}}
$$

is a manifestly topological term, and where the Futaki term

$$
\mathcal{B}(\Omega)=\frac{1}{32 \pi^{2}}\|\mathfrak{F}(\Omega)\|^{2}
$$

will eventually be shown to be uniformly bounded.

In practice, $\mathcal{A}$ is an explicitly computable rational function of several variables. Nonetheless, the actual expression is sufficiently complicated that the judicious use of computer algebra is of enormous help in reliably obtaining the correct answer. For this reason, a number of the proofs presented here partially depend on calculations carried out with the assistance of Mathemat$i c a$. However, these calculations are merely elaborate algebraic manipulations which could, in principle, be directly verified by a careful human with sufficient time and patience. For clarity of presentation, we have grouped these computer-assisted calculations into two appendices. In spite of their typographical location, these appendices are logically independent on the rest of the paper, and might rightly be said to represent the beginning rather than the end of the article. For this reason, results proved in the appendices are freely cited throughout the body of the paper, without the slightest danger of circular reasoning.

\section{Extremal Kähler Metrics}

In this section, we will prove two results on the existence of extremal Kähler metrics. While these appear [11] to be of genuinely independent interest, we will be principally interested in them here because of the key role they will play in the proofs of Theorems $\mathrm{A}$ and $\mathrm{B}$.

Theorem 1 Let $M \approx \mathbb{C P}_{2} \# 2 \overline{\mathbb{C P}}_{2}$ be the blow-up of $\mathbb{C P}_{2}$ at two distinct points, and let $[\omega]$ be a Kähler class on $M$ for which

$$
\mathcal{T}([\omega]):=\frac{\left(c_{1} \cdot[\omega]\right)^{2}}{[\omega]^{2}} \leq \frac{3}{2} c_{1}^{2}-\frac{1}{4}=c_{1}^{2}+3.25 .
$$

Then there is an extremal Kähler metric $g$ on $M$ with Kähler form $\omega \in[\omega]$. 
Theorem 2 Let $M \approx \mathbb{C P}_{2} \# 3 \overline{\mathbb{C P}}_{2}$ be the blow-up of $\mathbb{C P}_{2}$ at three noncollinear points, and let $[\omega]$ be a Kähler class on $M$ for which

$$
\mathcal{T}([\omega]):=\frac{\left(c_{1} \cdot[\omega]\right)^{2}}{[\omega]^{2}} \leq \frac{3}{2} c_{1}^{2}-\frac{1}{4}=c_{1}^{2}+2.75 .
$$

Then there is an extremal Kähler metric $g$ on $M$ with Kähler form $\omega \in[\omega]$.

To prove these results, we will rely on a continuity method argument analogous to the one used to prove [7, Theorem 27]. This time, however, we will start at the anti-canonical class $c_{1}$ and work outward. To make this feasible, we will temporarily just assume that $c_{1}$ is represented by an extremal Kähler metric. For $M=\mathbb{C P}_{2} \# 3 \overline{\mathbb{C P}}_{2}$, this is assumption is certainly valid, since the anti-canonical class class contains the Kähler-Einstein metric of Siu [24]. In the case of $M=\mathbb{C P}_{2} \# 2 \overline{\mathbb{C P}}_{2}$, we will eventually validate this assumption by providing a new proof in Proposition 1 below; in the interim, however, we will try to put the reader at ease by mentioning that this fact has actually been previously proved elsewhere [7, 13] using different methods.

By rescaling, we may also assume that the target Kähler class $[\omega]$ satisfies $c_{1} \cdot[\omega]=c_{1}^{2}$, so that $[\omega]=c_{1}+\eta$ for some $\eta \in H^{2}(M, \mathbb{R})$ with $c_{1} \cdot \eta=0$. We now join $c_{1}$ to the given $[\omega]$ by a straight line segment

$$
[0,1] \ni t \longmapsto\left[\omega_{t}\right]:=(1-t) c_{1}+t[\omega]=c_{1}+t \eta
$$

and notice that the $\left[\omega_{t}\right]$ are all Kähler classes, by convexity of the Kähler cone. Since $\eta^{2}<0$,

$$
\left[\omega_{t}\right]^{2}=\left(c_{1}+t \eta\right)^{2}=c_{1}^{2}+t^{2} \eta^{2} \geq c_{1}^{2}+\eta^{2}=[\omega]^{2},
$$

so that

$$
\mathcal{T}\left(\left[\omega_{t}\right]\right)=\frac{\left(c_{1} \cdot\left[\omega_{t}\right]\right)^{2}}{\left[\omega_{t}\right]^{2}} \leq \frac{\left(c_{1} \cdot[\omega]\right)^{2}}{[\omega]^{2}}=\mathcal{T}([\omega]) \leq \frac{3}{2} c_{1}^{2}-\frac{1}{4} \quad \forall t \in[0,1] .
$$

We are therefore required to prove the existence of a solution in each $\left[\omega_{t}\right]$. It is therefore natural to consider the set

$$
\mathcal{E}=\left\{t \in[0,1] \mid\left[\omega_{t}\right] \text { is represented by an extremal Kähler metric }\right\},
$$

and define

$$
\mathfrak{t}=\sup \{t \in[0,1] \mid[0, t] \subset \mathcal{E}\} .
$$


We have already assumed that $0 \in \mathcal{E}$, so $\mathcal{E} \neq \varnothing$, and $\mathfrak{t} \in[0,1]$. On the other hand, an inverse-function theorem argument [18 implies that $\mathcal{E}$ is open in $[0,1]$. One connected component of $\mathcal{E}$ therefore either takes the form $[0, \mathfrak{t})$ or $[0,1]$. It therefore suffices to show that $\mathfrak{t} \in \mathcal{E}$, as this will then immediately imply that $\mathcal{E}=[0,1]$.

To attain this goal, we will make systematic use of the weak compactness theorem of Chen and Weber [9]. This result guarantees that, given a sequence of unit-volume extremal Kähler metrics on a compact complex surface, one can extract a subsequence which Gromov-Hausdorff converges to an extremal Kähler orbifold metric, provided there is a uniform upper bound on the Sobolev constants. Such an upper bound can in turn be guaranteed [7] if the metrics in question have uniformly bounded, positive scalar curvature, and if all belong to the controlled cone

$$
\mathcal{A}([\omega])<\frac{3}{2} c_{1}^{2}-\epsilon
$$

for some $\epsilon>0$, where

$$
\mathcal{A}=\frac{1}{32 \pi^{2}} \int_{M} s^{2} d \mu
$$

for an extremal Kähler metric. However,

$$
\mathcal{A}=\mathcal{T}+\mathcal{B}
$$

where

$$
\mathcal{B}=\frac{1}{32 \pi^{2}} \int_{M}\left(s-s_{0}\right)^{2} d \mu
$$

for an extremal Kähler metric, and we show in Lemmas A.1 and B.1 that

$$
\mathcal{B}<\frac{1}{4}
$$

for every Kähler class on either of these manifolds. On either of these manifolds, it follows that the inequality (2) holds for any convergent sequence of Kähler classes with

$$
\mathcal{T} \leq \frac{3}{2} c_{1}^{2}-\frac{1}{4}
$$

where $\epsilon$ is the infimum of $\frac{1}{4}-\mathcal{B}$ over a small neighborhood of the limit class. Moreover, Lemmas A.2 and B.2 show that extremal Kähler metrics on these manifolds always have everywhere-positive scalar curvature which is 
uniformly bounded on any compact subset of the Kähler cone $\mathcal{K}$. Hence, by rescaling to unit volume and then scaling back, every sequence in $\mathcal{E}$ has a subsequence for which the corresponding extremal Kähler manifolds $\left\{\left(M, g_{j}\right)\right\}$ converge to an extremal Kähler orbifold $\left(N, g_{\infty}\right)$ in the Gromov-Hausdorff topology. Of course, the Kähler classes $\left[\omega_{j}\right]$ may simultaneously be taken to converge to some Kähler class $\Omega \in\left\{\left[\omega_{t}\right] \mid t \in(0,1]\right\}$.

We will now specialize to the case of an increasing sequence $t_{j} \nearrow \mathfrak{t}$, with the goal of showing that $\mathfrak{t} \in \mathcal{E}$. In order to show that, modulo diffeomorphisms, the $g_{j}$ actually converge smoothly to a metric on the given $M$, we must rule out bubbling. Recall [7, 9] that smooth convergence will fail only if the sectional curvatures of our metrics $g_{j}$ fail to be uniformly bounded, and that when this happens, after once again passing to a subsequence, one can find a sequence of rescalings $\kappa_{j}^{-1} g_{j}, \kappa_{j} \rightarrow 0$, and a sequence of base-points $p_{j} \in M$ such that $\left\{\left(M, p_{j}, \kappa_{j}^{-1} g_{j}\right)\right\}$ converges in the pointed Gromov-Hausdorff topology to a non-trivial ALE scalar-flat Kähler surface $\left(X, \hat{g}_{\infty}\right)$. Such a pointed limit is called a deepest bubble. Because all the metrics in our sequence are toric, so is the deepest bubble. This implies [7, Lemma 17] that $b_{2}(X) \neq 0$, and that $H_{2}(X, \mathbb{Z})$ is generated by embedded holomorphic $\mathbb{C P}_{1}$ 's. Moreover, for large $j$ in the subsequence, the pointed Gromov-Hausdorff convergence guarantees that $X$ is diffeomorphic to an open subset $U_{j} \subset M$, in such a manner that $c_{1}(X)$ obtained by restricting $c_{1}(M)$ to $U$, and such that $H_{2}\left(U_{j}\right)$ is generated by embedded 2-spheres $S_{j}$ which are symplectic with respect to the Kähler form $\omega_{j}$. Finally, the homomorphism $H_{2}\left(U_{j}, \mathbb{Z}\right) \rightarrow H_{2}(M, \mathbb{Z})$ induced by inclusion is injective, and the restriction of the intersection form of $M$ to $U_{j}$ is negative definite.

Our strategy will now combine ideas from [7] and [8]. Suppose that $\left(X, \hat{g}_{\infty}\right)$ is a deepest bubble arising from some sequence $g_{j}:=g_{t_{j}}$, where $t_{j} \nearrow \mathfrak{t}$. Let $S \subset X$ be any holomorphic embedded $\mathbb{C P}_{1}$, and let $k>0$ be the positive integer defined by $S \cdot S=-k$. Then for each $j$ sufficiently far out in our subsequence, we can find an $\omega_{t_{j}}$-symplectic 2-sphere $S_{j} \subset M$ with $S_{j} \cdot S_{j}=-k$, for some fixed positive integer $k$. By the adjunction formula, we would then also have $c_{1} \cdot S_{j}=2-k$. As $j$ varies, the homology class $\left[S_{j}\right]$ could in principle change. However, since $c_{1}^{2}>0$ and $b_{+}(M)=1$, the subset of $H_{2}(M, \mathbb{R})$ defined by

$$
\begin{aligned}
& c_{1} \cdot A=2-k \\
& A \cdot A=-k
\end{aligned}
$$


is compact, and the set of $A \in H_{2}(M, \mathbb{Z})$ satisfying these conditions is therefore finite. By refining our subsequence, we may therefore assume that $\left[S_{j}\right]=[S]$ is independent of $j$. Moreover, since $S$ has finite area in $\left(X, \hat{g}_{\infty}\right)$, which is a rescaled limit of regions $U_{j} \subset M$, with magnification tending to infinity, we must be able to represent $[S]$ by symplectic 2 -spheres $S_{j}$ of arbitrarily small area in $\left(M, g_{j}\right)$ as $j \rightarrow \infty$. Since the area of $S_{j}$ is $\geq\left|\left[\omega_{j}\right] \cdot\left[S_{j}\right]\right|$ by Wirtinger's inequality, taking the limit as $j \rightarrow \infty$ now yields

$$
\Omega \cdot A=0,
$$

where $\Omega=\left[\omega_{t}\right]$ is the limit Kähler class.

On the other hand, the sphere $S_{j}$ is symplectic with respect to each Kähler form $\omega_{j}=\omega_{t_{j}}$ in our subsequence. Now, by construction,

$$
\left[\omega_{j}\right]=u_{j} c_{1}+\left(1-u_{j}\right) \Omega
$$

for a sequence of positive numbers $u_{j}=1-\left(t_{j} / \mathfrak{t}\right) \searrow 0$. Since $S_{j}$ is symplectic, we therefore have $\left[\omega_{j}\right] \cdot A=\left[\omega_{j}\right] \cdot[S]>0$, for large $j$. Since $\Omega \cdot A=0$, this says that $u_{j}\left(c_{1} \cdot A\right)=\left[u_{j} c_{1}+\left(1-u_{j}\right) \Omega\right] \cdot A>0$. Hence

$$
c_{1} \cdot A>0 .
$$

However, $c_{1} \cdot A=2-k$ by the adjunction formula. We thus conclude that $k<2$. It follows that $k=1$, thereby reducing our bubbling problem to a single case.

To deal with the remaining $k=1$ case, we now classify the homology classes $A \in H_{2}(M, \mathbb{Z})$ satisfying

$$
\begin{aligned}
& c_{1} \cdot A=1 \\
& A \cdot A=-1 .
\end{aligned}
$$

For this purpose, it is best to concentrate on the case of $M=\mathbb{C P}_{2} \# 3 \overline{\mathbb{C P}}_{2}$, since we can identify $H^{2}\left(\mathbb{C P}_{2} \# 2 \overline{\mathbb{C P}}_{2}\right)$ with a hyperplane in $H^{2}\left(\mathbb{C P}_{2} \# 3 \overline{\mathbb{C P}}_{2}\right)$. If we choose a basis for $H_{2}\left(\mathbb{C P}_{2} \# 3 \overline{\mathbb{C P}}_{2}\right)$ consisting of a projective line $L$ and three exceptional divisors $E_{1}, E_{2}, E_{3}$, corresponding to the three blown-up points in $\mathbb{C P}_{2}$, the intersection form then becomes

$$
\left(\begin{array}{llll}
1 & & & \\
& -1 & & \\
& & -1 & \\
& & & -1
\end{array}\right)
$$


and $c_{1}$ is Poincaré dual to $(3,-1,-1,-1)$. Setting $A=(n, a, b, c)$, we thus have

$$
\begin{aligned}
3 n+a+b+c & =1 \\
n^{2}-a^{2}-b^{2}-c^{2} & =-1
\end{aligned}
$$

and it therefore follows that

$5\left(a^{2}+b^{2}+c^{2}\right)+(a-b)^{2}+(a-c)^{2}+(b-c)^{2}+(a+1)^{2}+(b+1)^{2}+(c+1)^{2}=13$.

In particular, $a^{2}+b^{2}+c^{2}<3$. On the other hand, $a^{2}+b^{2}+c^{2}=n^{2}+1 \geq 1$. Thus, after possibly permuting $E_{1}, E_{2}$, and $E_{3}$, we may assume that $c=0$, that $|a|=1$, and that $|b| \leq 1$. It is then easy to check that, again modulo permutations of the $E_{j}$, the only solutions are

$$
(n, a, b, c)=(0,1,0,0) \text { and }(1,-1,-1,0)
$$

respectively corresponding to

$$
A=E_{1} \text { and } A=L-E_{1}-E_{2} \text {. }
$$

Throwing in permutations, we conclude that there are exactly six possibilities

$$
A=E_{1}, E_{2}, E_{3}, L-E_{1}-E_{2}, L-E_{1}-E_{3}, L-E_{2}-E_{3}
$$

on $M=\mathbb{C P}_{2} \# 3 \overline{\mathbb{C P}}_{2}$, and exactly three possibilities

$$
A=E_{1}, E_{2}, L-E_{1}-E_{2}
$$

on $M=\mathbb{C P}_{2} \# 2 \overline{\mathbb{C P}}_{2}$. This is good news, because these classes are actually all represented by holomorphic (-1)-curves on either choice of $M$. Since these holomorphic curves must have positive area for any Kähler metric, any Kähler class $\Omega$ on $M$ must therefore satisfy $\Omega \cdot A>0$ for any such class $A=[S]$. This rules out bubbling when $k=1$, and our previous argument therefore shows that bubbling has now been definitively ruled out in all cases.

As $t_{j} \rightarrow \mathfrak{t}$, the sectional curvatures of the $g_{j}$ therefore remain uniformly bounded, and these metrics therefore converge to a smooth, toric, extremal Kähler metric on a complex surface diffeomorphic to $M$. The collection of totally geodesic holomorphic curves consisting of the points of non-trivial isotopy must converge to a configuration of totally geodesic holomorphic curves 
with the same self-intersections as the original curves in $M$, and with areas obtained by taking naïve limits, allowing us to read off both the limit complex structure and the limit Kähler class. This shows that the limit extremal Kähler metric is actually compatible with the original complex structure on $M$, with Käher class $\Omega$. Thus $\mathfrak{t} \in \mathcal{E}$, and hence $\mathcal{E}=[0,1]$. The target Kähler class $[\omega]$ therefore contains an extremal Kähler metric, and Theorems 1 and 2 have therefore been proved, modulo the assumption that $c_{1}$ contains an extremal Kähler metric. We now complete the argument by justifying this last assumption.

Proposition 1 The anti-canonical class of $\mathbb{C P}_{2} \# 2 \overline{\mathbb{C P}}_{2}$ is represented by an extremal Kähler metric.

Proof. We begin by once again recalling [24, 26] that $c_{1}$ is represented on $\mathbb{C P}_{2} \# 3 \overline{\mathbb{C P}}_{2}$ by a Kähler-Einstein metric; thus, we can safely apply the above arguments to $\mathbb{C P}_{2} \# 3 \overline{\mathbb{C P}}_{2}$ without assuming anything about $\mathbb{C P}_{2} \# 2 \overline{\mathbb{C P}}_{2}$. We now identify $\mathbb{C P}_{2} \# 2 \overline{\mathbb{C P}}_{2}$ with the blow-down of of $\mathbb{C P}_{2} \# 3 \overline{\mathbb{C P}}_{2}$ along the exceptional divisor $E_{1}$, and let

$$
\text { p : } \mathbb{C P}_{2} \# 3 \overline{\mathbb{C P}}_{2} \rightarrow \mathbb{C P}_{2} \# 2 \overline{\mathbb{C P}}_{2}
$$

denote the blowing-down map. If $\Omega$ is any Kähler class on $\mathbb{C P}_{2} \# 2 \overline{\mathbb{C P}}_{2}$, then

$$
\left[\omega_{t}\right]=(1-t) c_{1}+t\left(\mathbf{p}^{*} \Omega\right)
$$

is a Kähler class on $M=\mathbb{C P}_{2} \# 3 \overline{\mathbb{C P}}_{2}$ for any $t \in[0,1)$, and this Kähler class satisfies $\mathcal{T}\left(\left[\omega_{t}\right]\right) \leq \mathcal{T}(\Omega)$ for any $t$, since the Poincaré dual of $c_{1}\left(\mathbb{C P}_{2} \# 2 \overline{\mathbb{C P}}_{2}\right)$ is exactly the push-forward, via $\mathbf{p}$, of the Poincaré dual of $c_{1}\left(\mathbb{C P}_{2} \# 3 \overline{\mathbb{C P}}_{2}\right)$. We now assume henceforth that $\mathcal{T}(\Omega)<8.75$; this holds in particular, if we take $\Omega=c_{1}\left(\mathbb{C P}_{2} \# 2 \overline{\mathbb{C P}}_{2}\right)$, but we shall also allow for other possibilities, as doing so will cost us no additional effort.

Since $\mathcal{T}\left(\left[\omega_{t}\right]\right) \leq \mathcal{T}(\Omega)<8.75$, there is, by Theorem 2 , an extremal Kähler metric $g_{t}$ on $M$ with Kähler form $\omega_{t} \in\left[\omega_{t}\right]$ for all $t \in[0,1)$. Moreover, $\left[\omega_{t}\right]$ satisfies (2) for all $t \in[0,1)$, with $\epsilon=8.75-\mathcal{T}(\Omega)$. Because the $g_{t}$ also have positive, uniformly bounded scalar curvatures by Lemma B.2, these metrics therefore have uniformly bounded Sobolev constants, and the ChenWeber theorem therefore guarantees the existence of a Gromov-Hausdorff limit of some sequence $g_{t_{j}}, t_{j} \nearrow 1$, with limit a compact extremal Kähler orbifold $\left(N, J_{\infty}, g_{\infty}\right)$. On the other hand, the sectional curvatures of the 
$g_{t}$ are are certainly not uniformly bounded as $t \rightarrow 1$, as the presence of a totally geodesic 2 -sphere of area $\alpha=1-t \searrow 0$, forces $\sup K \nearrow+\infty$ by the classical Gauss-Bonnet theorem. Thus, a non-trivial deepest bubble must arise. On the other hand, our symplectic argument to rule out the bubbles containing a spherical class $A$ with $A^{2}=-k$ still works for $k \geq 2$; the only difference is that the limit class $\mathbf{p}^{*} \Omega \in H^{2}(M, \mathbb{R})$ no longer belongs to the Kähler cone, but rather sits on its boundary. We thus have still excluded any deepest bubble except one whose homology is carried by $(-1)$ curves. Moreover, $E_{1}$ is the only homological (-1)-curve whose symplectic area tends to zero as $t \rightarrow 1$. Since $E_{1}$ has non-zero self-intersection, it cannot be simultaneously arise from two disjoint bubbles, or from a bubble on a bubble. Thus, the limit orbifold must obtained from $M=\mathbb{C P}_{2} \# 3 \overline{\mathbb{C P}}_{2}$ by collapsing a single 2 -sphere representing $E_{1}$. Since the link of such a 2 -sphere is simply connected, it follows that the $N$ is a manifold, with $b_{2}=3$; similarly, the bubble has $b_{2}=1$, and is asymptotically Euclidean, rather than merely being ALE. Consequently, the bubble that forms must be the Burns metric [14] on $\mathcal{O}(-1)$ line bundle over $\mathbb{C P}_{1}$, since [15, 23], up to homothety, this is the only asymptotically Euclidean scalar-flat Kähler surface with $b_{2}=1$.

Because the Burns metric has isometry group $U(2)$, the toric structure of the bubble is therefore uniquely determined up to conjugation, with points of non-trival isotropy given by the zero section and two fibers of the line bundle. By contrast, the set of points of $M=\mathbb{C P}_{2} \# 3 \overline{\mathbb{C P}}_{2}$ at which the torus action has non-trivial isotropy consists of the six $(-1)$-curves

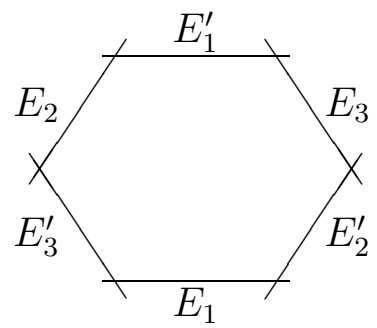

where $E_{j}^{\prime}-E_{j}=L-E_{1}-E_{2}-E_{3}$. Since the bubble is obtained by rescaling a tubular neighborhood of some 2-sphere representing $E_{1}$, and because the Killing fields generating its toric structure are limits of the Killing fields on $\mathbb{C P}_{2} \# 3 \overline{\mathbb{C P}}_{2}$, the rescaled region must in fact contain the exceptional divisor $E_{1}$, as this curve is one connected component of the zero locus of an appropriate Killing field. Since the Riemannian diameter of the region of curvature concentration tends to zero [9], and since radial geodesics in the 
Burns metric are length minimizing, even at large radii, the region of curvature concentration can only contain a disk of small intrinsic diameter in the curves $E_{2}^{\prime}$ and $E_{3}^{\prime}$; thus, the region of curvature concentration meets of the locus of exceptional isotropy only in $E_{1}$ and in small adjoining disks in $E_{2}^{\prime}$ and $E_{3}^{\prime}$. In particular, $E_{2}, E_{3}$, and $E_{1}^{\prime}$ are contained in the region of smooth convergence as $t_{j} \nearrow 1$. These totally geodesic submanifolds therefore give rise to totally geodesic submanifolds in the Gromov-Hausdorff limit. These limit submanifolds are moreover holomorphic curves, since the original complex structures converge smoothly to the limit complex structure $J_{\infty}$ in the region in question; and these three limit curves all have self-intersection -1 , since a tubular neighborhood of each original curve survives diffeomorphically in the limit. Thus, $\left(N, J_{\infty}\right)$ is a compact complex surface which can be blown down, at the limit $E_{2}$ and $E_{3}$ curves, to a compact complex surface with $b_{2}=1$ containing a rational curve of self-intersection +1 . Surface classification [2, Proposition 4.3] now tells us that this blow-down must be $\mathbb{C P}_{2}$. Hence $\left(N, J_{\infty}\right)$ is actually the blow-up of $\mathbb{C P}_{2}$ at two distinct points. Moreover, $\left(N, J_{\infty}, g_{\infty}\right)$ contains a chain of three $(-1)$-curves whose homology classes generate $H_{2}(N, \mathbb{R})$, and the areas of these curves are $\Omega\left(E_{2}\right), \Omega\left(E_{1}^{\prime}\right)$, and $\Omega\left(E_{3}\right)$. The limit extremal Kähler metric $g_{\infty}$ therefore has Kähler class $\Omega$. Specializing to the case where $\Omega$ is the anti-canonical class then proves the claim.

Notice that the above argument actually proves more than what was initially claimed. We therefore also have the following result:

Proposition 2 Let $\Omega$ be any Kähler class on $\mathbb{C P}_{2} \# 2 \overline{\mathbb{C P}}_{2}$ for which

$$
\mathcal{T}(\Omega)<8.75=c_{1}^{2}+1.75
$$

Then there is an extremal Kähler metric $g$ in $\Omega$, and a one-parameter family $g_{t}, t \in[0,1)$ of extremal Kähler metrics on $\mathbb{C P}_{2} \# 3 \overline{\mathbb{C P}}_{2}$, with $g_{0}$ is KählerEinstein, and with $g_{t_{j}} \rightarrow g$ in the Gromov-Hausdorff sense for some $t_{j} \nearrow 1$. 


\section{Einstein Metrics}

Let $(M, J)$ now be a Del Pezzo surface, and again let $\mathcal{K} \subset H^{2}(M, \mathbb{R})$ be its Kähler cone. The following variational principle [7, 16] unlocks the mysteries of conformally Kähler, Einstein metrics:

Proposition 3 Suppose that $h$ is an Einstein metric on $M$ which is conformally related to a $J$-compatible Kähler metric $g$ with Kähler class $[\omega] \in \mathcal{K}$. Then $[\omega]$ is a critical point of $\mathcal{A}$. Moreover, $g$ is an extremal Kähler metric, and the scalar curvature $s$ of $g$ is everywhere positive.

Conversely, if $\Omega \in \mathcal{K}$ is a critical point of $\mathcal{A}$, and if $\omega \in \Omega$ is the Kähler form of an extremal Kähler metric $g$ with scalar curvature $s>0$, then $h=$ $s^{-2} g$ is an Einstein metric on $M$.

Because of the crucial role this result plays in our discussion, we will now briefly sketch the proof, while referring the reader to [7] for more details.

For any smooth compact oriented 4-manifold $M$, one may consider the conformally invariant Riemannian functional

$$
\mathcal{W}(g)=\int_{M}|W|_{g}^{2} d \mu_{g}=-12 \pi^{2} \tau(M)+2 \int_{M}\left|W_{+}\right|^{2} d \mu
$$

where $W$ is the Weyl curvature, and $W_{+}$is its self-dual part. Following standard convention, we will call $\mathcal{W}$ the Weyl functional. The gradient of $\mathcal{W}$ on the space of RIemannian metrics [1, 3] is minus the Bach tensor B, as defined by

$$
\begin{aligned}
B_{a b} & =\left(\nabla^{c} \nabla^{d}+\frac{1}{2} r^{c d}\right) W_{a c b d} \\
& =\left(2 \nabla^{c} \nabla^{d}+r^{c d}\right)\left(W_{+}\right)_{a c b d} .
\end{aligned}
$$

Because $\int|W|^{2} d \mu$ is invariant under diffeomorphisms and conformal rescalings, we have

$$
B_{a}^{a}=0, \quad \nabla^{a} B_{a b}=0
$$

for any 4-dimensional Riemannian metric. The Bianchi identities imply that any Einstein metric satisfies the Bach-flat condition $B=0$. Since the Bach-flat condition is conformally invariant, any conformally Einstein 4-dimensional metric is therefore [3, 22] Bach-flat, too. 
We now specialize to the case of Kähler metrics. For any Kähler metric $g$ on a complex surface $(M, J)$, one has

$$
\left|W_{+}\right|^{2}=\frac{s^{2}}{24}
$$

with respect to the orientation induced by $J$. (Of course, equation (3) is not conformally invariant — but neither is the Kähler condition!) In particular, any Bach-flat Kähler metric is a critical point of the Calabi functional

$$
\mathcal{C}(g)=\int s^{2} d \mu
$$

either as a functional on a fixed Kähler class $\Omega=[\omega]$, or on the entire space of Kähler metrics, with $\Omega$ allowed to vary. In particular, any Bach-flat Kähler metric $g$ must be an extremal Kähler metric, and its Kähler class must be a critical point of $\mathcal{A}: \mathcal{K} \rightarrow \mathbb{R}$.

Equation (3) reflects the deeper fact that the self-dual Weyl tensor of a Kähler surface is completely determined by the scalar curvature and the Kähler form. If $\left(M^{4}, g, J\right)$ is a Kähler manifold with Kähler form $\omega$, then

$$
\left.\left(W_{+}\right)_{a b}{ }^{c d}=\frac{s}{12}\left[\omega_{a b} \omega^{c d}-\delta_{a}^{[c} \delta_{b}^{d]}+J_{a}^{[c} J_{b} d\right]\right]
$$

and plugging this into the formula for the Bach tensor yields

$$
B_{a b}=\frac{s}{6} \stackrel{\circ}{r}_{a b}+\frac{1}{4} J_{a}^{c} J_{b}{ }^{d} \nabla_{c} \nabla_{d} s+\frac{1}{12} \nabla_{a} \nabla_{b} s+\frac{1}{12} g_{a b} \Delta s .
$$

However, the extremal Kähler metric condition is equivalent to requiring that the Hessian $\nabla \nabla s$ of the scalar curvature by $J$-invariant. At an extremal Kähler metric, we can therefore define an anti-self-dual 2-form $\psi$ by

$$
\psi=B(J \cdot, \cdot)=\frac{1}{6}[s \rho+2 i \partial \bar{\partial} s]_{0}
$$

where $\rho$ is the Ricci form, and where the subscript "0" denotes projection into the primitive $(1,1)$-forms $\Lambda_{0}^{1,1}=\Lambda^{-}$. Since $B$ is symmetric and divergencefree, we thus have

$$
(\delta \psi)_{b}=-\nabla^{a} \psi_{a b}=\nabla^{a}\left(B_{a c} J_{b}^{c}\right)=J_{b}^{c} \nabla^{a} B_{a c}=0,
$$

so that the anti-self-dual 2-form $\psi$ is co-closed, and hence harmonic. In particular, the 1-parameter family $g_{t}=g+t B$ then consists entirely of 
Kähler metrics. The first variation $\mathcal{W}$ is $-\int|B|^{2} d \mu$ for this family of Kähler metrics, and so can vanish only if $B=0$. Thus, if $\Omega$ is a Kähler class which is a critical point of $\mathcal{A}$, and it $g$ is an extremal Kähler metric which belongs to $\Omega$, then $g$ is necessarily Bach-flat.

Now the trace-free Ricci tensor $\stackrel{\circ}{r}$ transforms under conformal changes by

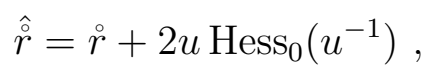

and equation (4) tells us that any Bach-flat Kähler metric satisfies

$$
\stackrel{\circ}{r}=-2 s^{-1} \operatorname{Hess}_{0}(s) .
$$

Thus, any Bach-flat Kähler metric with $s>0$ has a conformal rescaling $\hat{g}=s^{-2} g$ which is Einstein. Proposition 3 now follows.

Now let $M$ specifcally be the Del Pezzo surfaces $\mathbb{C P}_{2} \# 2 \overline{\mathbb{C P}}_{2}$, let $\mathcal{K} \subset$ $H^{2}(M, \mathbb{R})$ be its Kähler cone, and let $\check{\mathcal{K}}=\mathcal{K} / \mathbb{R}^{+}$, where the positive reals act by scalar multiplication. Since the function $\mathcal{T}(\Omega)=\left(c_{1} \cdot \Omega\right)^{2} / \Omega^{2}$ is homogeneous of degree 0 , we now consider it as a function on $\check{\mathcal{K}}$. For any $t \in \mathbb{R}$, let $\mathbf{Y}_{t} \subset \check{\mathcal{K}}$ be the region defined by $\mathcal{T}(\Omega) \leq t$.

Lemma 3.1 If $7<t<8$, then $\mathbf{Y}_{t}$ is homeomorphic to the closed unit 2-disk, and so, in particular, is compact.

Proof. On the manifold $M=\mathbb{C P}_{2} \# \overline{\mathbb{C P}}_{2}$ under discussion, an element of $H^{2}(M, \mathbb{R})$ is determined by the numbers

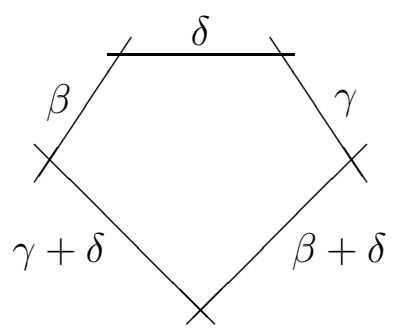

it assigns to the three exceptional curves portrayed as representing the upper edges of the pentagon. Since these three numbers represent areas, they all must be positive. Conversely, any cohomology class for which these three numbers are positive is actually a Kähler class. Indeed, we can either blow down $M$ along the top curve to obtain $\mathbb{C P}_{1} \times \mathbb{C P}_{1}$, or along the two top diagonal curves to obtain $\mathbb{C P}_{2}$. Pulling back products metrics on $\mathbb{C P}_{1} \times \mathbb{C P}_{1}$ 
then allows one to specify any desired value of $\beta$, and $\gamma$. Pulling back a multiple of the Fubini-Study metric and adding this on as well, we can then choose $\delta$ arbitrarily. Hence a cohomology class $\Omega \in H^{2}(M, \mathbb{R})$ is a Kähler class iff it assigns a positive value to each of these three exceptional curves. Of course, any cohomology class $\Omega$ with this property must consequently also satisfy $\Omega^{2}>0$ and $c_{1} \cdot \Omega>0$.

Now, suppose that the convex cone in $H^{2}(M, \mathbb{R})$ defined by

$$
\begin{aligned}
\Omega^{2} & >0 \\
c_{1}(M) \cdot \delta & >0 \\
\left(c_{1} \cdot \delta\right)^{2} & <8 \Omega^{2}
\end{aligned}
$$

contained a cohomology class that was not a Kähler class. By continuity, it would therefore contain a class $\Omega$ which was non-negative on all three exceptional curves, but which vanished on at least one of them. But $\Omega$ would then be the pull-back of some cohomology class $\mho$ on a blow-down $N$, obtained by collapsing exactly one exceptional curve, which satisfied $\mho^{2}>0$ and

$$
\left(c_{1}(N) \cdot \mho\right)^{2}<8 \mho^{2}=c_{1}^{2}(N) \mho^{2} .
$$

But this is a contradiction, because $c_{1}(N)$ and $\mho \in H^{2}(N, \mathbb{R})$ would then be a pair of time-like vectors in a 2-dimensional Minkowski space which violated the reverse Cauchy-Schwartz inequality for the Lorentzian inner product. Hence the open convex cone defined by (5) is actually a subset of the Kähler cone $\mathcal{K}$. Consequently, for any $t \in(7,8)$, the set of $\Omega \in H^{2}(M, \mathbb{R})$ with

$$
\begin{aligned}
\Omega^{2} & >0 \\
c_{1}(M) \cdot \Omega & >0 \\
\left(c_{1} \cdot \Omega\right)^{2} & \leq t \Omega^{2}
\end{aligned}
$$

consists entirely of Kähler classes, and its quotient by $\mathbb{R}^{+}$therefore exactly equals $\mathbf{Y}_{t}$. However, this quotient can be identified with the intersection of (6) with the hyperplane $c_{1} \cdot \Omega=7$. Writing elements of this hyperplane uniquely as $\Omega=c_{1}+\eta$, where $c_{1} \cdot \eta=0$, we thus have identified $\mathbf{Y}_{t}$ with the closed ball

$$
\left|\eta^{2}\right| \leq \frac{7(t-7)}{t}
$$

in the 2-dimensional space-like hyperplane $c_{1}^{\perp} \subset H^{2}(M, \mathbb{R})$. This proves the claim. 
Theorem $\mathrm{A}$ is now an easy consequence. Indeed, because Lemma A.1 tells us that $0 \leq \mathcal{B}<\frac{1}{4}$ on the entire Kähler cone, the infimum of $\mathcal{A}$ for $M=\mathbb{C P}_{2} \# 2 \overline{\mathbb{C P}}_{2}$ must be less than $\mathcal{T}\left(c_{1}\right)+\frac{1}{4}=c_{1}^{2}+\frac{1}{4}=7 \frac{1}{4}$, whereas $\mathcal{A} \geq 7 \frac{1}{4}$ outside the interior of $\mathbf{Y}_{c_{1}^{2}+\frac{1}{4}}$. Since $\mathbf{Y}_{c_{1}^{2}+\frac{1}{4}}$ is compact by Lemma 3.1. there is consequently an interior point $\check{\Omega}$ of $\mathbf{Y}_{c_{1}^{2}+\frac{1}{4}}$ at which $\mathcal{A}$ achieves its minimum. Notice that $\check{\Omega}$ is a critical point of $\mathcal{A}$, and let $\Omega$ be a Kähler class which projects to $\check{\Omega}$. By Theorem $1, \Omega$ is represented by an extremal Kähler metric $g$, and by Lemma A.2, this extremal Kähler metric has positive scalar curvature $s>0$. Proposition 3 then tells us that $h=s^{-2} g$ is an Einstein metric on $M$, and, by construction, $h$ minimizes the Weyl functional among all conformally Kähler metrics on $M$. We have thus succeeded in proving Theorem [A. Theorem B now similarly follows from Proposition 2, since $7.25<8.75$. 


\section{Appendix A: Computations for $\mathbb{C P} \# 2 \overline{\mathbb{C P}}_{2}$}

In this section, we will use a combination of elementary symplectic geometry and computer-assisted algebra to estimate some key geometric invariants of extremal Kähler metrics on $M=\mathbb{C P} \# 2 \overline{\mathbb{C P}}_{2}$ that are needed in the body of the paper. We begin by fixing a Kähler class, normalized by rescaling so that the proper transform of the projective line between the two blow-up points has area 1:
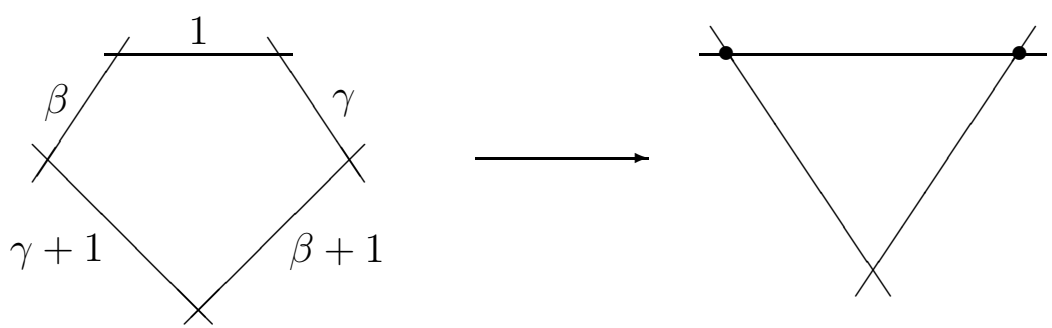

Take the two blow-up points to be $[1,0,0],[0,1,0] \in \mathbb{C P}_{2}$, and fix the maximal torus

$$
\left[\begin{array}{ccc}
e^{i \theta} & & \\
& e^{i \phi} & \\
& & 1
\end{array}\right]
$$

in the automophism group. Then, for any $T^{2}$-invariant metric, the moment map of the torus action will take values in a pentagon, which after translation becomes the following:

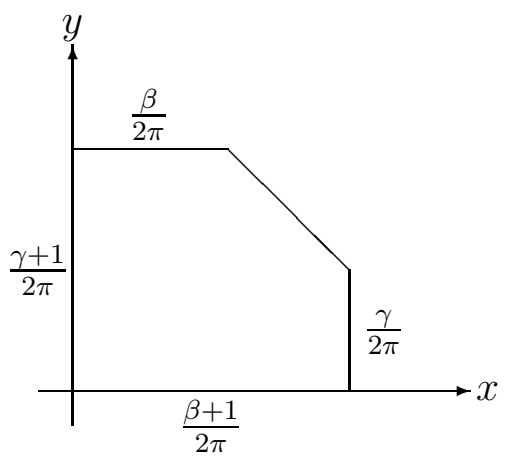

Let $\mathfrak{F}_{1}$ and $\mathfrak{F}_{2}$ be Futaki invariants of this Kähler class with respect to the vector fields with Hamiltonians $-x$ and $-y$. Then [19] for any $T^{2}$-invariant 
metric,

$$
\begin{aligned}
\mathfrak{F}_{1} & =\int_{M} x\left(s-s_{0}\right) d \mu \\
& =\frac{1}{V}\left[(\beta-2 \gamma)\left(\frac{1}{3}+\gamma+\gamma^{2}\right)+\gamma(\gamma-\beta)(2+\beta+2 \gamma)\right] \\
\mathfrak{F}_{2} & =\int_{M} y\left(s-s_{0}\right) d \mu \\
& =\frac{1}{V}\left[(\gamma-2 \beta)\left(\frac{1}{3}+\beta+\beta^{2}\right)+\beta(\beta-\gamma)(2+\gamma+2 \beta)\right]
\end{aligned}
$$

where

$$
V=\beta \gamma+\beta+\gamma+\frac{1}{2}
$$

Note that, by Archimedes' principle, the push-forward of the volume measure of $M$ is exactly $4 \pi^{2}$ times the Euclidean measure on the moment polygon. Thus, for example, the average values $x_{0}$ and $y_{0}$ of the Hamiltonians $x$ and $y$ on $M$ are also the $x$ and $y$ coordinates of the barycenter of the moment pentagon. This same observation also makes it straightforward to compute the following useful constants:

$$
\begin{aligned}
A & :=\int_{M}\left(x-x_{0}\right)^{2} d \mu \\
& =\frac{1+6(1+\beta)\left[\beta+\beta^{2}+\beta^{3}+\gamma\left(1+4 \beta+4 \beta^{2}+2 \beta^{3}\right)+\gamma^{2}(1+\beta)^{3}\right]}{288 \pi^{2} V} \\
B & :=\int_{M}\left(y-y_{0}\right)^{2} d \mu \\
& =\frac{1+6(1+\gamma)\left[\gamma+\gamma^{2}+\gamma^{3}+\beta\left(1+4 \gamma+4 \gamma^{2}+2 \gamma^{3}\right)+\beta^{2}(1+\gamma)^{3}\right]}{288 \pi^{2} V} \\
C & :=\int_{M}\left(x-x_{0}\right)\left(y-y_{0}\right) d \mu \\
& =-\frac{1+6(1+\beta)(1+\gamma)(\beta+\gamma+3 \beta \gamma)}{576 \pi^{2} V}
\end{aligned}
$$

If our metric is extremal, we then have

$$
s-s_{0}=a\left(x-x_{0}\right)+b\left(y-y_{0}\right)
$$


where the constants $a$ and $b$ are given by

$$
\begin{aligned}
a & =\frac{B \mathfrak{F}_{1}-C \mathfrak{F}_{2}}{A B-C^{2}} \\
b & =\frac{A \mathfrak{F}_{2}-C \mathfrak{F}_{1}}{A B-C^{2}} .
\end{aligned}
$$

Consequently,

$$
\int_{M}\left(s-s_{0}\right)^{2} d \mu=\frac{B \mathfrak{F}_{1}^{2}-2 C \mathfrak{F}_{1} \mathfrak{F}_{2}+A \mathfrak{F}_{2}^{2}}{A B-C^{2}}
$$

for any extremal Kähler metric, and even without assuming the existence of an extremal Kähler metric our arguments therefore assign a prominent role to the quantity

$$
\begin{aligned}
\mathcal{B}(\Omega)= & \frac{1}{32 \pi^{2}} \frac{B \mathfrak{F}_{1}^{2}-2 C \mathfrak{F}_{1} \mathfrak{F}_{+} A \mathfrak{F}_{2}^{2}}{A B-C^{2}} \\
= & 8\left[\gamma^{2}\left(1+4 \gamma+6 \gamma^{2}+4 \gamma^{3}\right)+\right. \\
& \beta \gamma\left(-1+3 \gamma+18 \gamma^{2}+26 \gamma^{3}+16 \gamma^{4}\right)+ \\
& 2 \beta^{5}\left(2+8 \gamma+21 \gamma^{2}+33 \gamma^{3}+27 \gamma^{4}+9 \gamma^{5}\right)+ \\
& \beta^{2}\left(1+3 \gamma+27 \gamma^{2}+79 \gamma^{3}+89 \gamma^{4}+42 \gamma^{5}\right)+ \\
& \beta^{4}\left(6+26 \gamma+89 \gamma^{2}+168 \gamma^{3}+150 \gamma^{4}+54 \gamma^{5}\right)+ \\
& \left.\beta^{3}\left(4+18 \gamma+79 \gamma^{2}+173 \gamma^{3}+168 \gamma^{4}+66 \gamma^{5}\right)\right] / \\
& {\left[48 \beta^{6}(1+\gamma)^{6}+48 \beta^{5}(1+\gamma)^{3}\left(3+12 \gamma+14 \gamma^{2}+6 \gamma^{3}\right)+\right.} \\
& (1+2 \gamma)^{2}\left(1+8 \gamma+20 \gamma^{2}+24 \gamma^{3}+12 \gamma^{4}\right)+ \\
& 4 \beta^{4}(1+\gamma)^{2}\left(47+282 \gamma+573 \gamma^{2}+504 \gamma^{3}+180 \gamma^{4}\right)+ \\
& 4 \beta\left(3+33 \gamma+140 \gamma^{2}+306 \gamma^{3}+376 \gamma^{4}+252 \gamma^{5}+72 \gamma^{6}\right)+ \\
& 8 \beta^{2}\left(7+70 \gamma+270 \gamma^{2}+535 \gamma^{3}+592 \gamma^{4}+354 \gamma^{5}+90 \gamma^{6}\right)+ \\
& \left.8 \beta^{3}\left(17+153 \gamma+535 \gamma^{2}+963 \gamma^{3}+966 \gamma^{4}+522 \gamma^{5}+120 \gamma^{6}\right)\right]
\end{aligned}
$$

Lemma A.1 One has $\mathcal{B}<\frac{1}{4}$ throughout the Kahler cone of $\mathbb{C P} \# 2 \overline{\mathbb{C P}}_{2}$. 
Proof. Subtracting 4 times the numerator of the above expression from the denominator yields

$$
\begin{aligned}
& 1+12 \gamma+24 \gamma^{2}+8 \gamma^{3}-4 \gamma^{4}+16 \gamma^{5}+48 \gamma^{6}+48 \beta^{6}(1+\gamma)^{6}+ \\
& 16 \beta^{5}\left(1+31 \gamma+93 \gamma^{2}+129 \gamma^{3}+108 \gamma^{4}+60 \gamma^{5}+18 \gamma^{6}\right)+ \\
& 4 \beta\left(3+41 \gamma+116 \gamma^{2}+162 \gamma^{3}+168 \gamma^{4}+124 \gamma^{5}+72 \gamma^{6}\right)+ \\
& 8 \beta^{2}\left(3+58 \gamma+162 \gamma^{2}+219 \gamma^{3}+236 \gamma^{4}+186 \gamma^{5}+90 \gamma^{6}\right)+ \\
& 8 \beta^{3}\left(1+81 \gamma+219 \gamma^{2}+271 \gamma^{3}+294 \gamma^{4}+258 \gamma^{5}+120 \gamma^{6}\right)+ \\
& 4 \beta^{4}\left(-1+168 \gamma+472 \gamma^{2}+588 \gamma^{3}+561 \gamma^{4}+432 \gamma^{5}+180 \gamma^{6}\right) .
\end{aligned}
$$

Term by term, this is greater than $4\left(\gamma^{2}-\gamma^{4}+\gamma^{6}+\beta^{2}-\beta^{4}+\beta^{6}\right)>0$. Thus the denominator of our expression for $\mathcal{B}$ is more than four times larger than the corresponding numerator. Hence $\mathcal{B}<\frac{1}{4}$, as claimed.

The coefficient $a$ of equation (7) is explicitly given by

$$
\begin{aligned}
& -192 \pi^{2} \gamma\left[1+4 \gamma+6 \gamma^{2}+4 \gamma^{3}+6 \beta^{3}(1+\gamma)^{3}+2 \beta^{2}\left(6+18 \gamma+17 \gamma^{2}+6 \gamma^{3}\right)+\beta(7+21 \gamma+\right. \\
& \left.\left.22 \gamma^{2}+10 \gamma^{3}\right)\right] /\left[1+10 \gamma+36 \gamma^{2}+64 \gamma^{3}+60 \gamma^{4}+24 \gamma^{5}+24 \beta^{5}(1+\gamma)^{5}+12 \beta^{4}(1+\right. \\
& \gamma)^{2}\left(5+20 \gamma+23 \gamma^{2}+10 \gamma^{3}\right)+16 \beta^{3}\left(4+28 \gamma+72 \gamma^{2}+90 \gamma^{3}+57 \gamma^{4}+15 \gamma^{5}\right)+12 \beta^{2}(3+ \\
& \left.\left.24 \gamma+69 \gamma^{2}+96 \gamma^{3}+68 \gamma^{4}+20 \gamma^{5}\right)+2 \beta\left(5+45 \gamma+144 \gamma^{2}+224 \gamma^{3}+180 \gamma^{4}+60 \gamma^{5}\right)\right]
\end{aligned}
$$

and $b$ is given by the analogous expression with $\beta$ and $\gamma$ interchanged. In particular, both of these coefficients are always negative.

Lemma A.2 If $g$ is an extremal Kähler metric on $M=\mathbb{C P}_{2} \# 2 \overline{\mathbb{C P}}_{2}$, then the scalar curvature $s$ of $g$ is positive at every point of $M$. Moreover, there is a smooth function $f: \mathcal{K} \rightarrow \mathbb{R}$ such that $s_{\max }=f(\Omega)$ for any extremal Kähler metric.

Proof. Since $a$ and $b$ are negative, the values of $s_{0}+a\left(x-x_{0}\right)+b\left(y-y_{0}\right)$ at $(0,0)$ and $\left(\frac{\beta}{2 \pi}, \frac{\gamma}{2 \pi}\right)$ are certainly upper and lower bounds for $s$. Making the substitution

$$
s_{0}=4 \pi \frac{c_{1} \cdot \Omega}{V}=4 \pi \frac{3+2 \beta+2 \gamma}{\frac{1}{2}+\beta+\gamma+\beta \gamma}
$$

into the value at $\left(\frac{\beta}{2 \pi}, \frac{\gamma}{2 \pi}\right)$ gives us the positive lower bound 


$$
\begin{aligned}
s_{\min } \geq 24 \pi & {\left[(1+2 \gamma)\left(1+2 \gamma+2 \gamma^{2}\right)^{2}+8 \beta^{5}(1+\gamma)^{4}+\right.} \\
& 4 \beta^{4}\left(5+24 \gamma+40 \gamma^{2}+32 \gamma^{3}+13 \gamma^{4}+2 \gamma^{5}\right)+ \\
& 8 \beta^{3}\left(3+14 \gamma+25 \gamma^{2}+26 \gamma^{3}+16 \gamma^{4}+4 \gamma^{5}\right)+ \\
& 4 \beta^{2}\left(4+16 \gamma+33 \gamma^{2}+50 \gamma^{3}+40 \gamma^{4}+12 \gamma^{5}\right)+ \\
& \left.2 \beta\left(3+12 \gamma+32 \gamma^{2}+56 \gamma^{3}+48 \gamma^{4}+16 \gamma^{5}\right)\right] / \\
& {\left[1+10 \gamma+36 \gamma^{2}+64 \gamma^{3}+60 \gamma^{4}+24 \gamma^{5}+24 \beta^{5}(1+\gamma)^{5}+\right.} \\
& 12 \beta^{4}(1+\gamma)^{2}\left(5+20 \gamma+23 \gamma^{2}+10 \gamma^{3}\right)+ \\
& 16 \beta^{3}\left(4+28 \gamma+72 \gamma^{2}+90 \gamma^{3}+57 \gamma^{4}+15 \gamma^{5}\right)+ \\
& 12 \beta^{2}\left(3+24 \gamma+69 \gamma^{2}+96 \gamma^{3}+68 \gamma^{4}+20 \gamma^{5}\right)+ \\
& \left.2 \beta\left(5+45 \gamma+144 \gamma^{2}+224 \gamma^{3}+180 \gamma^{4}+60 \gamma^{5}\right)\right]
\end{aligned}
$$

while making the same substitution into the value at $(0,0)$ gives us a smooth function $f$ with $f(\Omega)=s_{\max }$ for any extremal Kähler metric, and the requirement that $f$ be homogeneous of degree -1 then specifies an appropriate smooth extension of $f$ to the entire Kähler cone. 


\section{Appendix B: Computations for $\mathbb{C P} \# 3 \overline{\mathbb{C P}}_{2}$}

We now carry out computations analogous to those in the previous appendix, but this time for $M=\mathbb{C P} \# 3 \overline{\mathbb{C P}}_{2}$. First recall that the general Kähler class on this manifold is determined by four real numbers:

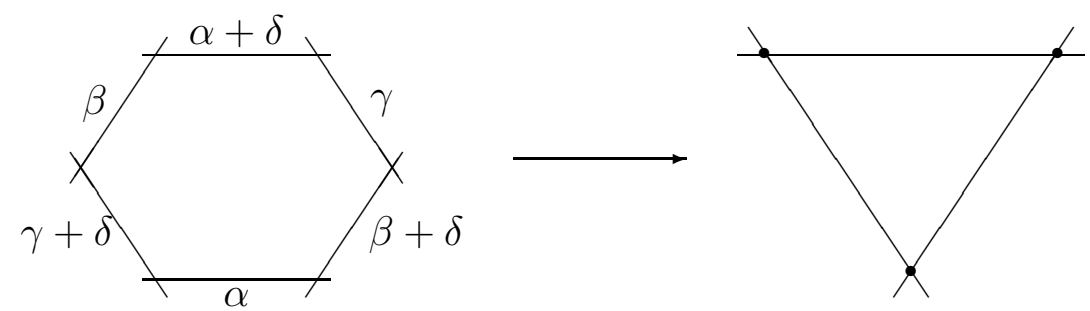

By applying a Cremona transformation, we may also assume that $\delta \geq 0$. After rescaling, the region $\delta>0$ can then be completely understood in terms of those classes for which $\delta=1$; these are exactly parameterized by the three arbitrary positive real numbers $\alpha, \beta$, and $\gamma$. Of course, any invariant geometrical conclusion we reach regarding this region will automatically also apply to "mirror" region reached by the Cremona transformation. This will allow us to understand the entire Kähler cone $\mathcal{K}$, as long as we are careful to also account for the hyperplane $\delta=0$.

We now once again fix the 2-torus in the automorphims group corresponding to $\left[z_{1}: z_{2}: z_{3}\right] \mapsto\left[e^{i \theta} z_{1}: e^{i \phi} z_{2}: z_{3}\right]$. The image of $M$ under the moment map is then the hexagon

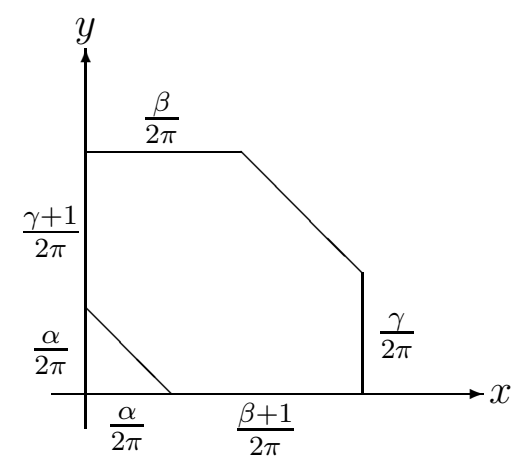


and our formulas [19] for the components of the Futaki invariant become

$$
\begin{aligned}
\mathfrak{F}_{1} & =\int_{M} x\left(s-s_{0}\right) d \mu \\
& =\frac{1}{V}\left[(\alpha+\beta-2 \gamma)\left(\frac{1}{3}+\gamma+\gamma^{2}\right)+(\gamma-\alpha)(\gamma-\beta)(2+\alpha+\beta+2 \gamma)\right] \\
\mathfrak{F}_{2} & =\int_{M} y\left(s-s_{0}\right) d \mu \\
& =\frac{1}{V}\left[(\alpha+\gamma-2 \beta)\left(\frac{1}{3}+\beta+\beta^{2}\right)+(\beta-\alpha)(\beta-\gamma)(2+\alpha+\gamma+2 \beta)\right]
\end{aligned}
$$

where

$$
V=\alpha \beta+\alpha \gamma+\beta \gamma+\alpha+\beta+\gamma+\frac{1}{2}
$$

is the volume of $(M, \Omega)$. Three other essential coefficients needed in our computation are

$$
\begin{aligned}
A= & \int_{M}\left(x-x_{0}\right)^{2} d \mu \\
= & \left(288 \pi^{2} V\right)^{-1}\left[1+6 \beta+12 \beta^{2}+12 \beta^{3}+6 \beta^{4}+6 \gamma^{2}(1+\beta)^{4}+6 \alpha^{4}(1+\gamma+\beta)^{2}+\right. \\
& 6 \gamma\left(1+5 \beta+8 \beta^{2}+6 \beta^{3}+2 \beta^{4}\right)+6 \alpha^{2}\left(2+8 \beta+9 \beta^{2}+4 \beta^{3}+\beta^{4}+6 \gamma^{2}(1+\beta)^{2}+\right. \\
& \left.2 \gamma(2+\beta)^{2}(1+2 \beta)\right)+12 \alpha^{3}\left(1+3 \beta+2 \beta^{2}+2 \gamma^{2}(1+\beta)+\gamma\left(3+6 \beta+2 \beta^{2}\right)\right)+ \\
& \left.6 \alpha\left(1+5 \beta+8 \beta^{2}+6 \beta^{3}+2 \beta^{4}+4 \gamma^{2}(1+\beta)^{3}+\gamma\left(5+20 \beta+24 \beta^{2}+12 \beta^{3}+2 \beta^{4}\right)\right)\right] \\
B:= & \int_{M}\left(y-y_{0}\right)^{2} d \mu \\
= & \left(288 \pi^{2} V\right)^{-1}\left[1+6 \gamma+12 \gamma^{2}+12 \gamma^{3}+6 \gamma^{4}+6 \beta^{2}(1+\gamma)^{4}+6 \alpha^{4}(1+\beta+\gamma)^{2}+\right. \\
& 6 \beta\left(1+5 \gamma+8 \gamma^{2}+6 \gamma^{3}+2 \gamma^{4}\right)+6 \alpha^{2}\left(2+8 \gamma+9 \gamma^{2}+4 \gamma^{3}+\gamma^{4}+6 \beta^{2}(1+\gamma)^{2}+\right. \\
& \left.2 \beta(2+\gamma)^{2}(1+2 \gamma)\right)+12 \alpha^{3}\left(1+3 \gamma+2 \gamma^{2}+2 \beta^{2}(1+\gamma)+\beta\left(3+6 \gamma+2 \gamma^{2}\right)\right)+ \\
& \left.6 \alpha\left(1+5 \gamma+8 \gamma^{2}+6 \gamma^{3}+2 \gamma^{4}+4 \beta^{2}(1+\gamma)^{3}+\beta\left(5+20 \gamma+24 \gamma^{2}+12 \gamma^{3}+2 \gamma^{4}\right)\right)\right]
\end{aligned}
$$


and

$$
\begin{aligned}
C:= & \int_{M}\left(x-x_{0}\right)\left(y-y_{0}\right) d \mu \\
= & -\left(576 \pi^{2} V\right)^{-1}\left[1+6 \gamma+6 \gamma^{2}+12 \alpha^{4}(1+\beta+\gamma)^{2}+6 \beta^{2}\left(1+4 \gamma+3 \gamma^{2}\right)+\right. \\
& 6 \beta\left(1+5 \gamma+4 \gamma^{2}\right)+24 \alpha^{3}\left(1+3 \gamma+2 \gamma^{2}+2 \beta^{2}(1+\gamma)+\beta\left(3+6 \gamma+2 \gamma^{2}\right)\right)+ \\
& 18 \alpha^{2}\left(1+4 \gamma+3 \gamma^{2}+\beta^{2}\left(3+6 \gamma+2 \gamma^{2}\right)+2 \beta\left(2+6 \gamma+3 \gamma^{2}\right)\right)+ \\
& \left.6 \alpha\left(1+5 \gamma+4 \gamma^{2}+2 \beta^{2}\left(2+6 \gamma+3 \gamma^{2}\right)+\beta\left(5+20 \gamma+12 \gamma^{2}\right)\right)\right]
\end{aligned}
$$

If our metric is extremal, we once again have

$$
s-s_{0}=a\left(x-x_{0}\right)+b\left(x-x_{0}\right)
$$

for the constants

$$
\begin{aligned}
a & =\frac{B \mathfrak{F}_{1}-C \mathfrak{F}_{2}}{A B-C^{2}} \\
b & =\frac{-C \mathfrak{F}_{1}+A \mathfrak{F}_{2}}{A B-C^{2}} .
\end{aligned}
$$

and hence

$$
\int_{M}\left(s-s_{0}\right)^{2} d \mu=\frac{B \mathfrak{F}_{1}^{2}-2 C \mathfrak{F}_{1} \mathfrak{F}_{+} A \mathfrak{F}_{2}^{2}}{A B-C^{2}}
$$

For any Kähler class $\Omega=[\omega]$ on $\mathbb{C P} \# 3 \overline{\mathbb{C P}}_{2}$ with $\delta=1, \mathcal{B}(\Omega)$ is the right-hand side over $32 \pi^{2}$, so automated calculation reveals that $\mathcal{B}(\Omega)$ equals

$$
\begin{aligned}
& {\left[\gamma^{2}\left(1+4 \gamma+6 \gamma^{2}+4 \gamma^{3}\right)+\beta \gamma\left(-1+3 \gamma+18 \gamma^{2}+26 \gamma^{3}+16 \gamma^{4}\right)+2 \beta^{5}\left(2+8 \gamma+21 \gamma^{2}+\right.\right.} \\
& \left.33 \gamma^{3}+27 \gamma^{4}+9 \gamma^{5}\right)+\beta^{2}\left(1+3 \gamma+27 \gamma^{2}+79 \gamma^{3}+89 \gamma^{4}+42 \gamma^{5}\right)+\beta^{4}\left(6+26 \gamma+89 \gamma^{2}+\right. \\
& \left.168 \gamma^{3}+150 \gamma^{4}+54 \gamma^{5}\right)+\beta^{3}\left(4+18 \gamma+79 \gamma^{2}+173 \gamma^{3}+168 \gamma^{4}+66 \gamma^{5}\right)+2 \alpha^{5}\left(2+9 \beta^{5}+\right. \\
& 8 \gamma+21 \gamma^{2}+33 \gamma^{3}+27 \gamma^{4}+9 \gamma^{5}+9 \beta^{4}(3+\gamma)+3 \beta^{2}(7+5 \gamma)+3 \beta^{3}(11+6 \gamma)+\beta(8+ \\
& \left.\left.12 \gamma+15 \gamma^{2}+18 \gamma^{3}+9 \gamma^{4}\right)\right)+\alpha^{4}\left(168 \gamma^{3}+150 \gamma^{4}+54 \gamma^{5}+18 \beta^{5}(3+\gamma)+\beta^{4}(150+\right. \\
& \left.72 \gamma-18 \gamma^{2}\right)+6 \beta^{3}\left(28+12 \gamma-15 \gamma^{2}-6 \gamma^{3}\right)+6+26 \gamma+89 \gamma^{2}+\beta^{2}\left(89-6 \gamma-162 \gamma^{2}-\right. \\
& \left.\left.90 \gamma^{3}-18 \gamma^{4}\right)+2 \beta\left(13+2 \gamma-3 \gamma^{2}+36 \gamma^{3}+36 \gamma^{4}+9 \gamma^{5}\right)\right)+\alpha^{2}\left(1+3 \gamma+27 \gamma^{2}+\right. \\
& 79 \gamma^{3}+89 \gamma^{4}+42 \gamma^{5}+6 \beta^{5}(7+5 \gamma)-\beta^{4}\left(-89+6 \gamma+162 \gamma^{2}+90 \gamma^{3}+18 \gamma^{4}\right)+3 \beta^{2}(9- \\
& \left.56 \gamma-165 \gamma^{2}-144 \gamma^{3}-54 \gamma^{4}\right)-\beta^{3}\left(-79+111 \gamma+432 \gamma^{2}+324 \gamma^{3}+90 \gamma^{4}\right)+3 \beta(1- \\
& \left.\left.23 \gamma-56 \gamma^{2}-37 \gamma^{3}-2 \gamma^{4}+10 \gamma^{5}\right)\right)+\alpha^{3}\left(4+18 \gamma+79 \gamma^{2}+173 \gamma^{3}+168 \gamma^{4}+66 \gamma^{5}+\right.
\end{aligned}
$$




$$
\begin{aligned}
& 6 \beta^{5}(11+6 \gamma)+6 \beta^{4}\left(2812 \gamma-15 \gamma^{2}-6 \gamma^{3}\right)+\beta^{3}\left(173-324 \gamma^{2}-216 \gamma^{3}-36 \gamma^{4}\right)- \\
& \left.\beta^{2}\left(-79+111 \gamma+432 \gamma^{2}+324 \gamma^{3}+90 \gamma^{4}\right)+\beta\left(18-46 \gamma-111 \gamma^{2}+72 \gamma^{4}+36 \gamma^{5}\right)\right)+ \\
& \alpha\left(2 \beta^{5}\left(8+12 \gamma+15 \gamma^{2}+18 \gamma^{3}+9 \gamma^{4}\right)+\gamma\left(-1+3 \gamma+18 \gamma^{2}+26 \gamma^{3}+16 \gamma^{4}\right)+2 \beta^{4}(13+\right. \\
& \left.2 \gamma-3 \gamma^{2}+36 \gamma^{3}+36 \gamma^{4}+9 \gamma^{5}\right)+3 \beta^{2}\left(1-23 \gamma-56 \gamma^{2}-37 \gamma^{3}-2 \gamma^{4}+10 \gamma^{5}\right)+\beta(-1- \\
& \left.\left.\left.30 \gamma-69 \gamma^{2}-46 \gamma^{3}+4 \gamma^{4}+24 \gamma^{5}\right)+\beta^{3}\left(18-46 \gamma-111 \gamma^{2}+72 \gamma^{4}+36 \gamma^{5}\right)\right)\right] \\
& {\left[( 1 + 2 \gamma + 2 \beta ( 1 + \gamma ) + 2 \alpha ( 1 + \beta + \gamma ) ) \left(1+10 \gamma+36 \gamma^{2}+64 \gamma^{3}+60 \gamma^{4}+24 \gamma^{5}+\right.\right.} \\
& 24 \beta^{5}(1+\gamma)^{5}+24 \alpha^{5}(1+\beta+\gamma)^{5}+12 \beta^{4}(1+\gamma)^{2}\left(5+20 \gamma+23 \gamma^{2}+10 \gamma^{3}\right)+16 \beta^{3}(4+ \\
& \left.28 \gamma+72 \gamma^{2}+90 \gamma^{3}+57 \gamma^{4}+15 \gamma^{5}\right)+12 \beta^{2}\left(3+24 \gamma+69 \gamma^{2}+96 \gamma^{3}+68 \gamma^{4}+20 \gamma^{5}\right)+ \\
& 2 \beta\left(5+45 \gamma+144 \gamma^{2}+224 \gamma^{3}+180 \gamma^{4}+60 \gamma^{5}\right)+12 \alpha^{4}(1+\beta+\gamma)^{2}\left(5+20 \gamma+23 \gamma^{2}+\right. \\
& \left.10 \gamma^{3}+10 \beta^{3}(1+\gamma)+\beta^{2}\left(23+46 \gamma+16 \gamma^{2}\right)+2 \beta\left(10+30 \gamma+23 \gamma^{2}+5 \gamma^{3}\right)\right)+16 \alpha^{3}(4+ \\
& 28 \gamma+72 \gamma^{2}+90 \gamma^{3}+57 \gamma^{4}+15 \gamma^{5}+15 \beta^{5}(1+\gamma)^{2}+3 \beta^{4}\left(19+57 \gamma+50 \gamma^{2}+13 \gamma^{3}\right)+ \\
& 3 \beta^{3}\left(30+120 \gamma+155 \gamma^{2}+78 \gamma^{3}+13 \gamma^{4}\right)+3 \beta^{2}\left(24+120 \gamma+206 \gamma^{2}+155 \gamma^{3}+50 \gamma^{4}+\right. \\
& \left.\left.5 \gamma^{5}\right)+\beta\left(28+168 \gamma+360 \gamma^{2}+360 \gamma^{3}+171 \gamma^{4}+30 \gamma^{5}\right)\right)+12 \alpha^{2}\left(3+24 \gamma+69 \gamma^{2}+\right. \\
& 96 \gamma^{3}+68 \gamma^{4}+20 \gamma^{5}+20 \beta^{5}(1+\gamma)^{3}+\beta^{4}\left(68+272 \gamma+366 \gamma^{2}+200 \gamma^{3}+36 \gamma^{4}\right)+ \\
& 4 \beta^{3}\left(24+120 \gamma+206 \gamma^{2}+155 \gamma^{3}+50 \gamma^{4}+5 \gamma^{5}\right)+2 \beta\left(12+84 \gamma+207 \gamma^{2}+240 \gamma^{3}+\right. \\
& \left.\left.136 \gamma^{4}+30 \gamma^{5}\right)+\beta^{2}\left(69+414 \gamma+864 \gamma^{2}+824 \gamma^{3}+366 \gamma^{4}+60 \gamma^{5}\right)\right)+2 \alpha\left(60 \beta^{5}(1+\right. \\
& \gamma)^{4}+12 \beta^{4}\left(15+75 \gamma+136 \gamma^{2}+114 \gamma^{3}+43 \gamma^{4}+5 \gamma^{5}\right)+12 \beta^{2}\left(12+84 \gamma+207 \gamma^{2}+\right. \\
& \left.240 \gamma^{3}+136 \gamma^{4}+30 \gamma^{5}\right)+8 \beta^{3}\left(28+168 \gamma+360 \gamma^{2}+360 \gamma^{3}+171 \gamma^{4}+30 \gamma^{5}\right)+5+45 \gamma+ \\
& \left.\left.144 \gamma^{2}+224 \gamma^{3}+180 \gamma^{4}+60 \gamma^{5}+3 \beta\left(15+120 \gamma+336 \gamma^{2}+448 \gamma^{3}+300 \gamma^{4}+80 \gamma^{5}\right)\right)\right]
\end{aligned}
$$

Lemma B.1 One has $\mathcal{B}<\frac{1}{4}$ throughout the Kahler cone of $\mathbb{C P} \# 3 \overline{\mathbb{C P}}_{2}$.

Proof. Subtracting four times the numerator from the denominator yields

$$
\begin{aligned}
& 1+12 \gamma+24 \gamma^{2}+8 \gamma^{3}-4 \gamma^{4}+16 \gamma^{5}+48 \gamma^{6}+48 \beta^{6}(1+\gamma)^{6}+48 \alpha^{6}(1+\beta+\gamma)^{6}+ \\
& 16 \beta^{5}\left(1+31 \gamma+93 \gamma^{2}+129 \gamma^{3}+108 \gamma^{4}+60 \gamma^{5}+18 \gamma^{6}\right)+4 \beta\left(3+41 \gamma+116 \gamma^{2}+\right. \\
& \left.162 \gamma^{3}+168 \gamma^{4}+124 \gamma^{5}+72 \gamma^{6}\right)+8 \beta^{2}\left(3+58 \gamma+162 \gamma^{2}+219 \gamma^{3}+236 \gamma^{4}+186 \gamma^{5}+\right. \\
& \left.90 \gamma^{6}\right)+8 \beta^{3}\left(1+81 \gamma+219 \gamma^{2}+271 \gamma^{3}+294 \gamma^{4}+258 \gamma^{5}+120 \gamma^{6}\right)+4 \beta^{4}(-1+168 \gamma+ \\
& \left.472 \gamma^{2}+588 \gamma^{3}+561 \gamma^{4}+432 \gamma^{5}+180 \gamma^{6}\right)+16 \alpha^{5}\left(1+31 \gamma+93 \gamma^{2}+129 \gamma^{3}+108 \gamma^{4}+\right. \\
& 60 \gamma^{5}+18 \gamma^{6}+18 \beta^{6}(1+\gamma)+12 \beta^{5}\left(5+16 \gamma+7 \gamma^{2}\right)+6 \beta^{4}\left(18+102 \gamma+98 \gamma^{2}+27 \gamma^{3}\right)+ \\
& 3 \beta^{3}\left(43+324 \gamma+482 \gamma^{2}+276 \gamma^{3}+54 \gamma^{4}\right)+3 \beta^{2}\left(31+275 \gamma+550 \gamma^{2}+482 \gamma^{3}+196 \gamma^{4}+\right. \\
& \left.\left.28 \gamma^{5}\right)+\beta\left(31+330 \gamma+825 \gamma^{2}+972 \gamma^{3}+612 \gamma^{4}+192 \gamma^{5}+18 \gamma^{6}\right)\right)+4 \alpha^{4}(-1+168 \gamma+ \\
& 472 \gamma^{2}+588 \gamma^{3}+561 \gamma^{4}+432 \gamma^{5}+180 \gamma^{6}+180 \beta^{6}(1+\gamma)^{2}+24 \beta^{5}\left(18+102 \gamma+98 \gamma^{2}+\right. \\
& \left.27 \gamma^{3}\right)+\beta^{4}\left(561+6468 \gamma+9624 \gamma^{2}+5112 \gamma^{3}+936 \gamma^{4}\right)+12 \beta^{3}\left(49+757 \gamma+1505 \gamma^{2}+\right. \\
& \left.1196 \gamma^{3}+426 \gamma^{4}+54 \gamma^{5}\right)+4 \beta\left(42+650 \gamma+1788 \gamma^{2}+2271 \gamma^{3}+1617 \gamma^{4}+612 \gamma^{5}+\right.
\end{aligned}
$$


$\left.\left.90 \gamma^{6}\right)+2 \beta^{2}\left(236+3576 \gamma+8631 \gamma^{2}+9030 \gamma^{3}+4812 \gamma^{4}+1176 \gamma^{5}+90 \gamma^{6}\right)\right)+4 \alpha(3+$ $41 \gamma+116 \gamma^{2}+162 \gamma^{3}+168 \gamma^{4}+124 \gamma^{5}+72 \gamma^{6}+72 \beta^{6}(1+\gamma)^{5}+4 \beta^{5}(31+330 \gamma+$ $\left.825 \gamma^{2}+972 \gamma^{3}+612 \gamma^{4}+192 \gamma^{5}+18 \gamma^{6}\right)+4 \beta^{4}\left(42+650 \gamma+1788 \gamma^{2}+2271 \gamma^{3}+\right.$ $\left.1617 \gamma^{4}+612 \gamma^{5}+90 \gamma^{6}\right)+\beta\left(41+570 \gamma+1812 \gamma^{2}+2816 \gamma^{3}+2600 \gamma^{4}+1320 \gamma^{5}+\right.$ $\left.360 \gamma^{6}\right)+2 \beta^{2}\left(58+906 \gamma+2832 \gamma^{2}+4189 \gamma^{3}+3576 \gamma^{4}+1650 \gamma^{5}+360 \gamma^{6}\right)+2 \beta^{3}(81+$ $\left.\left.1408 \gamma+4189 \gamma^{2}+5778 \gamma^{3}+4542 \gamma^{4}+1944 \gamma^{5}+360 \gamma^{6}\right)\right)+8 \alpha^{3}\left(1+81 \gamma+219 \gamma^{2}+\right.$ $271 \gamma^{3}+294 \gamma^{4}+258 \gamma^{5}+120 \gamma^{6}+120 \beta^{6}(1+\gamma)^{3}+6 \beta^{5}\left(43+324 \gamma+482 \gamma^{2}+276 \gamma^{3}+\right.$ $\left.54 \gamma^{4}\right)+6 \beta^{4}\left(49+757 \gamma+1505 \gamma^{2}+1196 \gamma^{3}+426 \gamma^{4}+54 \gamma^{5}\right)+\beta^{3}(271+5778 \gamma+$ $\left.14082 \gamma^{2}+14328 \gamma^{3}+7176 \gamma^{4}+1656 \gamma^{5}+120 \gamma^{6}\right)+\beta\left(81+1408 \gamma+4189 \gamma^{2}+\right.$ $\left.5778 \gamma^{3}+4542 \gamma^{4}+1944 \gamma^{5}+360 \gamma^{6}\right)+\beta^{2}\left(219+4189 \gamma+11592 \gamma^{2}+14082 \gamma^{3}+\right.$ $\left.\left.9030 \gamma^{4}+2892 \gamma^{5}+360 \gamma^{6}\right)\right)+8 \alpha^{2}\left(3+58 \gamma+162 \gamma^{2}+219 \gamma^{3}+236 \gamma^{4}+186 \gamma^{5}+90 \gamma^{6}+\right.$ $90 \beta^{6}(1+\gamma)^{4}+6 \beta^{5}\left(31+275 \gamma+550 \gamma^{2}+482 \gamma^{3}+196 \gamma^{4}+28 \gamma^{5}\right)+\beta^{4}(236+3576 \gamma+$ $\left.8631 \gamma^{2}+9030 \gamma^{3}+4812 \gamma^{4}+1176 \gamma^{5}+90 \gamma^{6}\right)+3 \beta^{2}\left(54+944 \gamma+2838 \gamma^{2}+3864 \gamma^{3}+\right.$ $\left.2877 \gamma^{4}+1100 \gamma^{5}+180 \gamma^{6}\right)+\beta\left(58+906 \gamma+2832 \gamma^{2}+4189 \gamma^{3}+3576 \gamma^{4}+1650 \gamma^{5}+\right.$ $\left.\left.360 \gamma^{6}\right)+\beta^{3}\left(219+4189 \gamma+11592 \gamma^{2}+14082 \gamma^{3}+9030 \gamma^{4}+2892 \gamma^{5}+360 \gamma^{6}\right)\right)$

Since this is term-by-term larger than

$$
4\left(\alpha^{2}-\alpha^{4}+\alpha^{6}\right)+4\left(\beta^{2}-\beta^{4}+\beta^{6}\right)+4\left(\gamma^{2}-\gamma^{4}+\gamma^{6}\right)>0,
$$

the denominator is more than four times larger than the numerator, and $\mathcal{B}<\frac{1}{4}$ on the complement of the hyperplane $\delta=0$ in the Kähler cone $\mathcal{K}$. Since the Futaki invariant vanishes on this hyperplane [19], $\mathcal{B}=0$ there, and we therefore have the strict inequality $\mathcal{B}<\frac{1}{4}$ on all of $\mathcal{K}$.

Lemma B.2 If $g$ an extremal Kähler metric on $M=\mathbb{C P}_{2} \# 3 \overline{\mathbb{C P}}_{2}$, then the scalar curvature $s$ of $g$ is positive everywhere on $M$. Moreover, there is a continuous function $f: \mathcal{K} \rightarrow \mathbb{R}$ such that $s_{\max }=f(\Omega)$ for any extremal Kähler metric, and this $f$ remains bounded as one approaches the pull-back of any class from $\mathbb{C P}_{2} \# 2 \overline{\mathbb{C P}}_{2}$.

Proof. The group of permutations of $\alpha, \beta$, and $\gamma$ acts transitively on the vertices of our hexagon, so it essentially suffices to compute the value of $s$ at a given vertex, since the maximum and minimum must occur at some critical point. In fact, evaluating $s$ at the vertex $(x, y)=(\alpha / 2 \pi, 0)$ gives

$24 \pi\left[1+10 \gamma+32 \gamma^{2}+48 \gamma^{3}+36 \gamma^{4}+8 \gamma^{5}+8 \beta^{5}(1+\gamma)^{4}+8 \alpha^{5}(1+\beta+\gamma)^{4}+4 \beta^{4}(9+\right.$
$\left.44 \gamma+80 \gamma^{2}+68 \gamma^{3}+25 \gamma^{4}+2 \gamma^{5}\right)+8 \beta^{3}\left(6+37 \gamma+80 \gamma^{2}+78 \gamma^{3}+34 \gamma^{4}+4 \gamma^{5}\right)+4 \beta^{2}(8+$ 


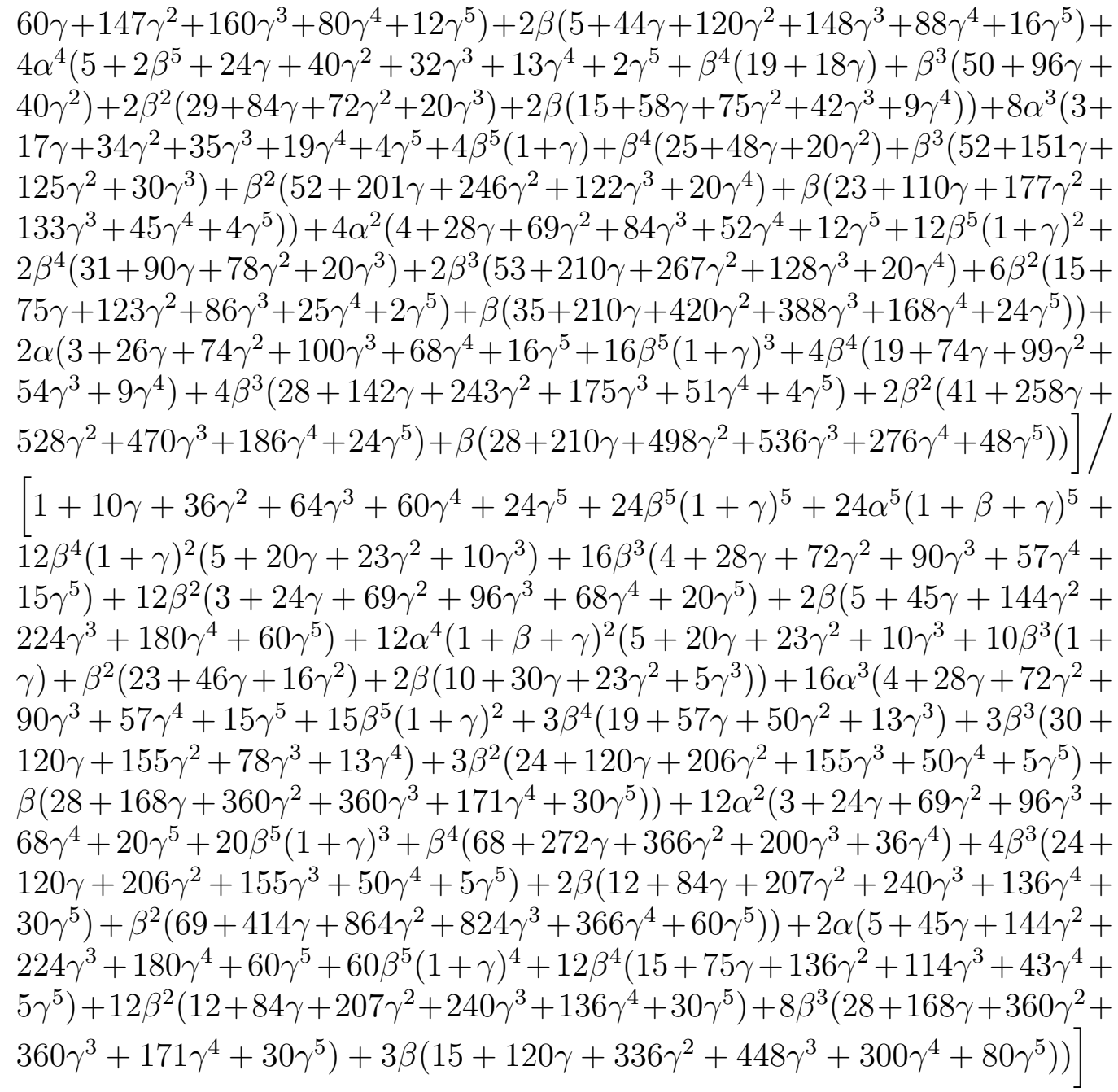

which is smooth and term-by-term positive for $\alpha, \beta, \gamma \geq 0$.

This expression can be uniquely extended to all $\delta>0$ by turning the numerator and denominator into homogeneous polynomials of $(\alpha, \beta, \gamma, \delta)$ of degree 9 and 10, respectively. The resulting expression is then smooth across $\delta=0$, because the numerator and denominator of the above expression actually do contain some terms of degree 9 and 10, respectively. Permuting $\alpha$, $\beta$, and $\gamma$, we obtain six smooth positive functions. Taking the minimum of these then shows that $s_{\min }$ is everywhere positive, while taking the maximum produces the required continuous positive function $f: \mathcal{K} \rightarrow \mathbb{R}$. 


\section{References}

[1] R. BACH, Zur Weylschen Relativitätstheorie und der Weylschen Erweiterung des Krümmungstensorbegriffs., Math. Zeitschr., 9 (1921), pp. 110-135.

[2] W. Barth, C. Peters, and A. Van De Ven, Compact complex surfaces, vol. 4 of Ergebnisse der Mathematik und ihrer Grenzgebiete (3), Springer-Verlag, Berlin, 1984.

[3] A. L. Besse, Einstein manifolds, vol. 10 of Ergebnisse der Mathematik und ihrer Grenzgebiete (3), Springer-Verlag, Berlin, 1987.

[4] E. Calabi, Extremal Kähler metrics, in Seminar on Differential Geometry, vol. 102 of Ann. Math. Studies, Princeton Univ. Press, Princeton, N.J., 1982, pp. 259-290.

[5] — Extremal Kähler metrics. II, in Differential Geometry and Complex Analysis, Springer, Berlin, 1985, pp. 95-114.

[6] X. X. Chen, Space of Kähler metrics. III. On the lower bound of the Calabi energy and geodesic distance, Invent. Math., 175 (2009), pp. 453503.

[7] X. X. Chen, C. LeBrun, And B. Weber, On conformally Kähler, Einstein manifolds, J. Amer. Math. Soc., 21 (2008), pp. 1137-1168.

[8] X. X. Chen and B. Wang, Kähler Ricci flow on Fano surfaces (I). e-print arXiv:0710.5204 [math.DG].

[9] X. X. Chen And B. Weber, Moduli spaces of critical Riemannian metrics with $L^{n / 2}$ norm curvature bounds. e-print arXiv:0705.4440, 2007.

[10] A. Derdziński, Self-dual Kähler manifolds and Einstein manifolds of dimension four, Compositio Math., 49 (1983), pp. 405-433.

[11] S. K. Donaldson, Extremal metrics on toric surfaces: a continuity method, J. Differential Geom., 79 (2008), pp. 389-432.

[12] A. Futaki And T. Mabuchi, Bilinear forms and extremal Kähler vector fields associated with Kähler classes, Math. Ann., 301 (1995), pp. 199-210. 
[13] W. He, Remarks on the existence of bilaterally symmetric extremal Kähler metrics on $\mathbb{C P}^{2} \# 2 \overline{\mathbb{C P}^{2}}$, Int. Math. Res. Not. IMRN, (2007). ID rnm127, 13 pages, doi:10.1093/imrn/rnm127.

[14] C. LeBrun, Counter-examples to the generalized positive action conjecture, Comm. Math. Phys., 118 (1988), pp. 591-596.

[15] _ Poon's self-dual metrics and Kähler geometry, J. Differential Geom., 28 (1988), pp. 341-343.

[16] _ Einstein metrics on complex surfaces, in Geometry and Physics (Aarhus, 1995), vol. 184 of Lecture Notes in Pure and Appl. Math., Dekker, New York, 1997, pp. 167-176.

[17] —, On Einstein, Hermitian 4-manifolds. e-print arXiv:1010.0238 [math.DG], 2010.

[18] C. LeBrun and S. R. Simanca, On the Kähler classes of extremal metrics, in Geometry and Global Analysis (Sendai, 1993), Tohoku Univ., Sendai, 1993, pp. 255-271.

[19] — Extremal Kähler metrics and complex deformation theory, Geom. Funct. Anal., 4 (1994), pp. 298-336.

[20] Y. Matsushima, Sur la structure du groupe d'homéomorphismes d'une certaine varieté Kählérienne, Nagoya Math. J., 11 (1957), pp. 145-150.

[21] D. PAGE, A compact rotating gravitational instanton, Phys. Lett., 79B (1979), pp. 235-238.

[22] R. Penrose and W. Rindler, Spinors and space-time. Vol. 2, Cambridge University Press, Cambridge, 1986. Spinor and twistor methods in space-time geometry.

[23] Y. S. Poon, Compact self-dual manifolds with positive scalar curvature, J. Differential Geom., 24 (1986), pp. 97-132.

[24] Y. Siu, The existence of Kähler-Einstein metrics on manifolds with positive anti-canonical line bundle and suitable finite symmetry group, Ann. Math., 127 (1988), pp. 585-627. 
[25] G. Tian, On Calabi's conjecture for complex surfaces with positive first Chern class, Inv. Math., 101 (1990), pp. 101-172.

[26] G. Tian and S. T. Yau, Kähler-Einstein metrics on complex surfaces with $\mathbf{c}_{1}>$ 0, Comm. Math. Phys., 112 (1987), pp. 175-203.

Acknowledgements. The author would like to warmly thank Xiuxiong Chen and Yuan Fang for their critical reading of an early draft of the manuscript, which resulted in substantial corrections, and eventually led to major simplifications and improvements.

AUTHOR'S ADDRESS:

Mathematics Department, SUNY, Stony Brook, NY 11794, USA

AUTHOR'S E-MAIL:

claude@math. sunysb.edu 